UCRL-JC-118972

PREPRINT

\title{
METALLIC MULTILAYERS AT THE NANOSCALE
}

\author{
A. F. Jankowski
}

This paper was prepared for submittal to NANO '94 Second Intemational Conference on Nanostructured Materials

in Stuttgart, Germany, on October 2-7, 1994.

This is a preprint of a paper intended for publication in a journal or proceedings. Since changes may be made before publication, this preprint is made available with the understanding that it will not be cited or reproduced without the permission of the author.

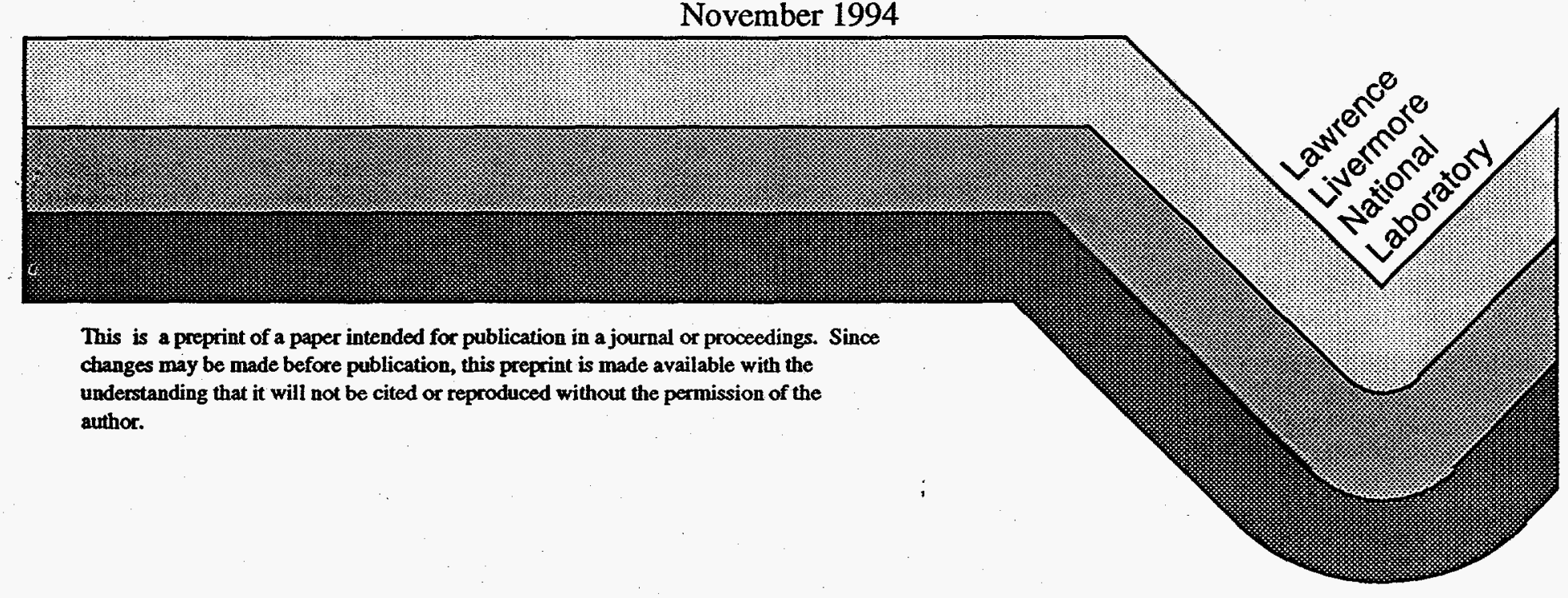


DISClaIMER

This document was prepared as an account of work sponsored by an agency of the United States Government. Neither the United States Government mor the University of Californin nor any of their employees, makes any warranty, express or implied, or assumes any legal liability or responsibility for the accuracy, completenesen, or mecfulness of any information, apparatus, product, or precess disclosed, or represents that its ase would not Infringe privately owned rights. Reference herein to any apecific commercial products, process, or service by trade nume, trademark, manufheturec, of ocherwise, does not necessarily constitute or imply its endorsement, recommendation, or favoring by the United States Government or the University of California. The views and opinions of authors expressed herein do not necessarily state or reflect those of the United States Government or the University of California, and shall not be used for advertising or product endorsement purposes. 


\section{DISCLAIMER}

Portions of this document may be illegible in electronic image products. Images are produced from the best available original document. 


\title{
METALLIC MULTILAYERS AT THE NANOSCALE
}

\author{
Alan F. Jankowski \\ University of California, Lawrence Livermore National Laboratory, \\ Department of Chemistry \& Materials Science, Livermore, CA 94550, USA
}

\begin{abstract}
The development of multilayer structures has been driven by a wide range of commercial applications requiring enhanced material behaviors. Innovations in physical vapor deposition technologies, in particular magnetron sputtering, have enabled the synthesis of metallic-based structures with nanoscaled layer dimensions as small as one-to-two monolayers. Parameters used in the deposition process are paramount to the formation of these small layer dimensions and the stability of the structure. Therefore, optimization of the desired material properties must be related to assessment of the actual microstructure. Characterization techniques as $x$-ray diffraction and high resolution microscopy are useful to reveal the interface and layer structure - whether ordered or disordered crystalline, amorphous, compositionally abrupt or graded, and/or lattice strained. Techniques for the synthesis of metallic multilayers with subnanometric layers will be reviewed with applications based on enhancing material behaviors as reflectivity and magnetic anisotropy but with emphasis on experimental studies of mechanical properties.
\end{abstract}

\section{INTRODUCTION}

The development of metallic multilayers at the nanoscale has evolved with synthesis technology and potential applications. The ability to reduce layer thicknesses to less than $1 \mathrm{~nm}$ in addition to controlling both growth morpholgy and interface structure has impacted many areas of application, as magneto-optical recording media and focusing $\mathrm{x}$-ray systems. The control of magnetic anisotropy through epitaxial growth strains is evidenced in results for the $\mathrm{Co} / \mathrm{Pt}, \mathrm{Fe} / \mathrm{Pt}, \mathrm{Co} / \mathrm{Cu}$ and $\mathrm{Co} / \mathrm{Pd}$ multilayer systems.(1-5) In each case, anisotropy is reported for samples with Co or Fe layers only 1-to-2 monolayers thick. Advances in the use of multilayers for hard $\mathrm{x}$-ray focusing has been shown feasible through the synthesis of $\mathrm{W} / \mathrm{B}_{4} \mathrm{C}$ with layers that are amorphous and only $0.33 \mathrm{~nm}$ thick.(6) Advantageous use of protective multilayer coatings, as $\mathrm{TiC} / \mathrm{TiB}_{2}$, for hard and wear resistant applications has been shown for high speed steel, cutting tools.(7) A review of the development of synthesis methods for producing multilayered coatings and films for the study of mechanical properties at nanoscale will follow with results of commonly used structural characterization techniques.

\section{SYNTHESIS}

The use of physical vapor deposition (PVD) techniques dominate the synthesis methods used to produce subnanometric layers. Even for the tool industry, the use of chemical vapor deposition to produce nitride and carbide coatings may gradually give way to PVD technologies. Amongst the many PVD methods used to produce multilayers, as electron beam 


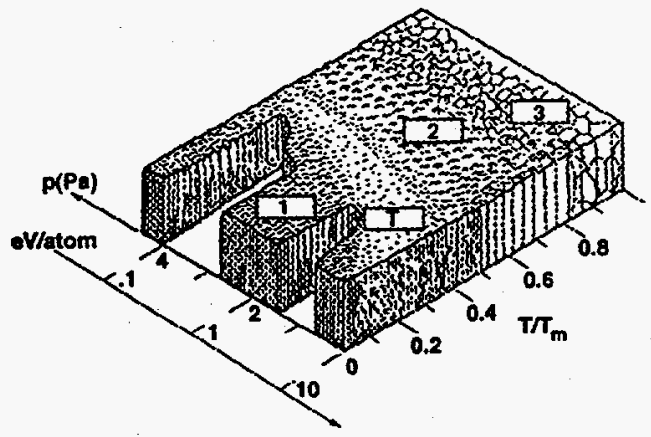

Fig. 1 - The zone diagram for morphology of vapor deposited films.(14)

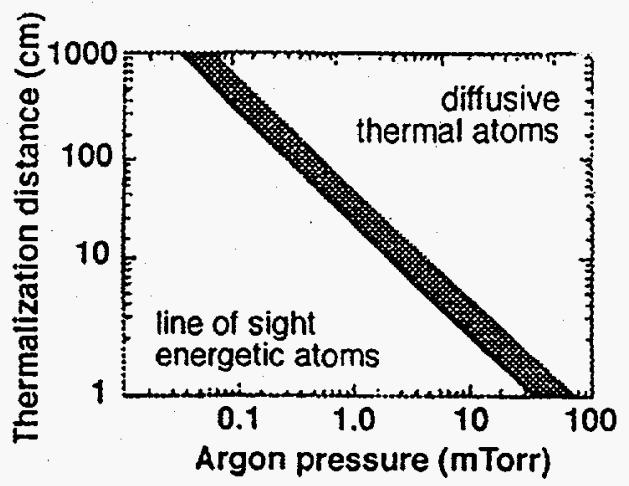

Fig.2 - The thermalization distance of sputtered neutrals varies with working gas pressure.(14)

evaporation, thermal evaporation, ion beam sputtering, and molecular beam epitaxy, the method used most frequently is magnetron sputtering. In dc or $\mathrm{rf}$ mode, with inert and/or reactive gases, magnetron sputtering offers great flexibility to coat both flat and curved surfaces with virtually every element of the periodic table.

\section{Deposition Energetics}

To facilitate the layer-by-layer growth mechanism at the subnanometric scale requires a precise control of the energetics of the deposition process. The sputtering process permits access to regulate the growth process through a large number of deposition parameters. For other PVD processes, as electron beam evaporation, deposition parameters can have competing effects on film growth structure. An example is found in the synthesis of $\mathrm{Mo} / \mathrm{Si}$ multilayers for $x$-ray projection lithography. (8-10) An increase in the substrate temperature enhances reflectivity by favoring smooth layering. However, interfacial diffusion is also enhanced which reduces reflectivity. Parameterization of the sputter deposition process includes working gas composition, pressure and flow, applied target voltage and current, source-to-substrate separation, as well as substrate conditioning, temperature and bias. The effect of changing only the Ar sputter gas pressure is seen in the measured, $x$-ray reflectivity of a W/C multilayer.(11) The efficiency $\left(R_{\text {meas }} / R_{\text {theo }}\right)$ decreases by an order of magnitude as the gas pressure is increased from $0.67 \mathrm{~Pa}(5.0 \mathrm{mTorr})$ to $1.67 \mathrm{~Pa}(12.5 \mathrm{mTorr})$. The reduction in reflectivity is attributed to an increase in interfacial roughness. The reason why the gas pressure change leads to this reduction is best related to the energetics of the deposition process.

The energy of the sputtered neutrals arriving at the growing film surface is perhaps most controllable through optimization of a just a few parameters - gas pressure and source-tosubstrate separation. At a sufficient separation, the substrate is not adversely effected by energy reflected from the target as carried by electrons, sputtered particles and reflected ions. The energy of the sputtered neutral is reduced by an order of magnitude or more from initial values of 10 to $30 \mathrm{eV} .(12,13)$ A dense columnar deposit is readily achieved at this energy level as seen in the zone diagram of film morphology (Fig. 1) adapted to include the influence of energy per deposited atom.(14) To avoid the interfacial intermixing effects from energetic neutrals ( $>3$ $\mathrm{eV})$ and the porosity of fully thermalized neutrals $(<0.1 \mathrm{eV})$, the pressure-distance product.(Fig. 2) can be used to fine tune the loss of energy of the sputtered neutrals attributable to scattering by the sputter gas.(13-15) Even for a basic model using kinetic gas theory (15), this approach 
has proven effective to minimize deposition induced amorphization of interfaces in Mo/Si x-ray mirrors and $\mathrm{Ni} / \mathrm{Ti}$ neutron optics.(16) The design of the magnetron sources also influences the flux of the energetic neutrals, in addition to the typical deposition process parameters.(17)

\section{Deposition Chambers}

The features of a deposition chamber which facilitate subnanometric layer formation incorporate the principals of discretely depositing materials at apriori controlled energetic levels. Substrates are mounted to a conformal table which is distanced from the deposition sources. The ultimate protection from particulate debris settling on the substrate surface is provided by an upward transport of the deposition flux. Sputtering is most flexible amongst deposition methods as it accomodates virtually any substrate-source geometry. The substrate table should be temperature controlled or permit an applied bias to help regulate surface bombardment/mobility. Ion-beam assisted deposition processes also supplement this objective of reconstructing the film surface during the growth process. For most metals, this can require the capability of effectively heating to $0.4 \mathrm{~T}_{\text {melt }}$. The source-to-substrate separation should be at least $10 \mathrm{~cm}$ for typical target voltages of 250 to 750 Volts in order to provide the conditons for near thermalization of the vapor. A stationary substrate requires shuttered deposition fluxes. A moving substrate provides greater thickness uniformity over larger areas than for a stationary substrate. A moving substrate also alleviates the need for a sequenced shuttering of sources.(18) Sources can be operated simultaneously providing each source is individually collimated along the line-of-sight to the substrate table. The gas pressure, composition and flow should each be independently adjustable for sputter and/or reactive deposition processes. Deposition rates are ideally monitored and controlled through a feedback loop to ensure uniformity of desired layer thickness over many layer pairs. To avoid contamination from atmospheric gases, a load lock system is ideal to shuttle samples under high vacuum. Chamber bake-outs with a residual gas analyzer to monitor contamination also suffices. These system features are basic to a multilayer synthesis chamber.

The sputter deposition system used to successively produce 1-to-2 monolayer thick alternating layers of $\mathrm{Fe}$ in $\mathrm{Fe}_{\mathrm{x}} \mathrm{Co}_{1-\mathrm{x}} / \mathrm{Pt}$ as well as W and $\mathrm{B}_{4} \mathrm{C}$ in W/B ${ }_{4} \mathrm{C}$ multilayers includes the following features. $(3,6,11)$ The chamber is cryogenically pumped to a base pressure of $9.0 \times 10^{-6} \mathrm{~Pa}$. The chamber is equipped with quartz lamps to provide a low temperature bake of system components to remove residual moisture and entrapped gases. A circular array of (3) planar magnetron sources are situated $20 \mathrm{~cm}$ beneath an oxygen-free $\mathrm{Cu}$ substrate table. A tungsten heater has been designed to water cool or heat the substrate table to temperatues of $500^{\circ} \mathrm{C}$ as monitored through thermocouples or an optical pyrometer. The substrate table can be rotated at a frequency, for actual depositions, that range from 0.03 to $4.0 \mathrm{~min}^{-1}$. As many as eight $5.1 \mathrm{~cm}$ diameter wafers can be coated uniformly (to within a $2 \%$ thickness variation) as each substrate passes sequentially over each sputtering target. One or two gases can be independently used for sputtering the $6.3 \mathrm{~cm}$ diameter targets. An argon (or other composition of inert and reactive gas) pressure range of 0.2 to $5 \mathrm{~Pa}$ is supplied at a mass flow rate of 5 to 50 $\mathrm{cc} \min ^{-1}$. The magnetron sources can be operated in the $\mathrm{dc}$ or $\mathrm{rf}$ mode with typical discharge voltages of 100 to 400 in either the power or current control modes. The instantaneous deposition rates are calibrated to the applied target power using $6 \mathrm{MHz}$ quartz crystal microbalance sensors. The deposition rates can be included in a feedback loop to the source power supplies to adjust sputtering rate or controlled by an operating program. This system has been operated continuously for as periods as long as $\mathbf{3 6}$ hours to produce multilayer films. 


\section{STRUCTURE}

\section{Characterization Methods}

The primary methods used to examine the structure of metallic multilayers are $\mathrm{x}$-ray diffraction (XRD) and transmission electron microscopy (TEM). XRD methods enable characterization of film texture, composition modulation and structural coherence, both in-plane and along the growth direction. A review of XRD methods to study and analize multilayer diffraction spectra is given elsewhere (19) which covers the use of double crystal diffractometry to the determine strain state as defined by inhomogeneous, gradients and periodic strain modulations. Grazing incidence as well as a symetrically transmitting geometry for XRD enables characterization parallel to the surface. TEM coupled with high resolution imaging in cross-section and selected area diffraction (SAD) provides an analysis of growth texture and morpholgy as well as lattice structure and distortions. In combination, a thorough structural characterization of the metallic multilayer is obtainable. The magnetron sputter deposition of $\mathrm{Mo} / \mathrm{V}$ is an example which represents both an understanding of the growth kinetics and the use of structural characterization.(20) In a study of potential application for hard and wear protective coatings, single crystal superlattices of $\mathrm{Mo} / \mathrm{V}$ were prepared by deposition on $\mathrm{MgO}(100)$ substrates heated to $700^{\circ} \mathrm{C}$. High resolution TEM was coupled with grazing incidence diffraction to reveal a microstructure for a $4.8 \mathrm{~nm}$ layer pair sample that was characterized by coherent modulation along the growth direction with coherent interfaces. $A \pm 1$ atomic layer interface width was modelled to the grazing incidence XRD spectra. Additional fitting procedures have been developed to determine both interdiffusion and roughness at superlattice interfaces from the damping of Fresnel oscillations between satellite reflections at grazing incidence.(21)

\section{Lattice Distortions}

Intrinsic to any interpretation of physical behavior is the underlying state of structural distortions. For semiconductor, strained layered superlattices, this characterization is routine but has only recently evolved with metallic multilayer phenomena. Early in the observation of biaxially-induced film stiffening (22) the need was recognized to precisely assess in-plane lattice parameters in order to distinguish the effects of interplanar spacing contractions from those caused by local concentration changes.(23) The measurement of in-plane lattice distortions has clearly lagged behind changes associated with the growth direction. A 5\% expansion of the Ni lattice associated with $\mathrm{C}_{44}$ softening of $\mathrm{Ni} / \mathrm{Mo}$ was recognized in modelling using molecular dynamics (24) but in-plane effects went unprobed.

Multilayer systems with a large atomic misfit, e.g. $15 \%$ in $\mathrm{Au} / \mathrm{Ni}$, should facilitate the measurement of in-plane strains characteristic to the film biaxial stress-state. TEM imaging coupled with microdiffraction of cross-sectioned Au/ $/$ i (111) superlattices revealed discrete differences in both the Au and Ni layers as compared to bulk properties. A $2 \%$ contraction of $\mathrm{Au}(220)$ and a $4 \%$ expansion of $\mathrm{Ni}(220)$ were measured from SAD patterns.(25) In-plane the multilayer film is polycrystalline with coherent grain sizes $<10 \mathrm{~nm}$ for the 50 layer pair, $2 \mathrm{~nm}$ modulation wavelength. A seed layer of $\mathrm{Au}$ (deposited at $150^{\circ} \mathrm{C}$ ) was used to initiate (111) texture in the multilayer (deposited at $50^{\circ} \mathrm{C}$ ) using electron beam evaporation. Later, in-plane lattice spacings for $\mathrm{Au} / \mathrm{Ni}$ multilayers prepared using the same preparation method were measured for a $0.8 \mathrm{~nm}$ modulation using XRD in a symmetrically transmitting geometry.(26) Complete in-plane lattice coherency was observed wherein the Ni layer had a $10 \%$ expansion and the Au layer a 5\% contraction (Fig. 3). 


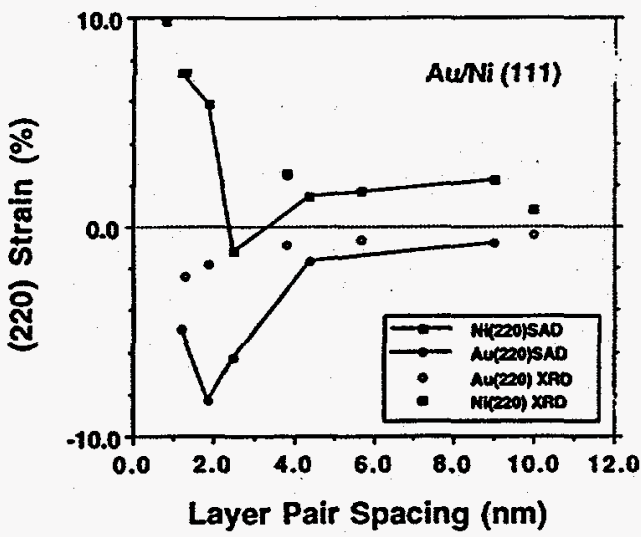

Fig. 3 - The in-plane strain measured in both $A u$ and Ni layers via $X R D(26)$ and $S A D(27)$.

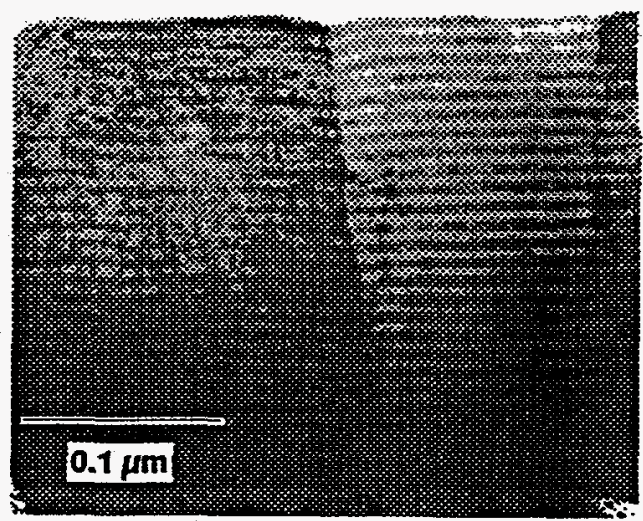

Fig.4 - A cross-section HREM image of a 9nm Au/Ni multilayer, sputter deposited film(27).

Results of a $10 \mathrm{~nm}$ grain size and in-plane lattice expansion > contraction for $\mathrm{Au} / \mathrm{Ni}$, totaling less than that required for complete misfit accomodation, are consistent with a low substrate temperature $\left(50^{\circ} \mathrm{C}\right)$ for an electron beam, multilayer deposition. Although the low substrate temperature will reduce interface diffusion, a low temperature will also produce highly defected structures without dense columnar grains. This structure is a typical result of the thermalized state of the evaporated species and the very low surface mobility for the room temperature substrate. Full misfit accomodation is more likely for a smaller modulation $(0.8 \mathrm{~nm})$. This proposed reasoning is consistent with the growth conditions and morphologies observed for electron beam deposits of $\mathrm{Mo} / \mathrm{Si}$.(8) Continuation of the low temperature, defected grain and columnar growth structure can be interupted by adding a greater number of interfaces per unit length along the growth direction. As a result, grain boundary discontinuities are substantially reduced within the film plane.

Full misfit accomodation to longer modulation wavelengths might be expected for a sputtering process with energetic neutrals. Greater surface mobility, hence a tendency for complete epitaxial-misfit accomodation should occur without complete thermalization of the sputtered neutrals. This case is found for sputtered Au/Ni as revealed through TEM (Fig. 4), HREM and SAD studies.(27) Rather than accomodate misfit through the primarily expansion mechanism of $\mathrm{Ni}$ (which is representative of a low temperature electron beam deposit), the greater mobility of the sputtered atoms creates a senario accessible to greater in-plane compression of the Au layer. In-plane SAD measurements show contraction of the Au layer equivalent to the expansion of the $\mathrm{Ni}$ layer for $1 \mathrm{~nm}$ modulations (Fig. 3). Like the electron beam evaporated multilayer films, the sputtered samples are (111) textured in the growth direction but polycrystalline in-plane. For a larger $(2 \mathrm{~nm})$ modulation wavelength, the total magnitude of misfit accomodation was reduced to $10 \%$, wherein the majority was of the Au layer in compression causing a net contraction for this layer pair spacing. Findings similar to those for the nearly thermalized sputtered deposits should be obtainable for an electron beam deposition provided a higher substrate temperature leads to greater surface mobility.

Large strain fluxuations periodic with the growth direction have been speculated for metallic multilayers with large lattice misfits (28) despite the small change in average lattice parameter measured along the growth diretion (29). The $\theta / 2 \theta$ XRD spectra of $\mathrm{Au} / \mathrm{Ni}$ multilayers have been modeled using both kinematical and dynamical diffraction theory to reveal the strain profile as associated with each atomic plane along the growth direction. $(30,31)$ 
The results indicate large periodic strains associated with the atomic planes adjacent to each Au$\mathrm{Ni}$ interface. The Au layers are in contraction and the Ni layers are in expansion along the growth direction. This finding shows a non-Poisson effect in the component layers, whereas the multilayer on average follows a Poisson relation.

The use of in-plane lattice spacing measurements has limitations for polycrystalline multilayer samples of material systems with identical crystal structures and mutual solubility. A question should be raised as to the quantitative accuracy of the strain values. Averaging effects from mutually reflecting grains of dissimilar ( $\mathrm{hkl}$ ) can result in broad diffraction peaks with overlapping reflections which make interpretation ambiguous. Clearly, single crystal spectra from either single crystals or single grains within a polycrystal (as can be found in SAD patterns) are more reliable for precise orientation and measurement of unambiguous in-plane spacings.

The results for lattice distortions in $\mathrm{Nb} / \mathrm{Cu}$ are representative for a class of metallic multilayers which are of different crystal structures and no mutual solubility.(32) Magnetron sputter deposition in the dc mode was used to produce $\mathrm{Nb}(110) / \mathrm{Cu}(111)$ textured growths on silicon substrates with in-plane polcrystallinity. Reflection XRD spectra $\left(\chi=0^{\circ}\right)$ revealed an increase in both the $\mathrm{Cu}$ and $\mathrm{Nb}$ spacings along the growth direction. In-plane, transmission XRD spectra $\left(\chi=90^{\circ}\right)$ revealed expanded $\mathrm{Cu}$ planes (a non-Poisson effect) and contracted Nb planes along both [002] and [110]. Analysis performed to determine the in-plane strains for $\mathrm{Nb} / \mathrm{Cu}$ fulfilled the requirement that the individual layers scatter incoherently along the layers. The magnitude of strain increased with decreasing layer pair spacings both along the growth direction, as found previously for $\mathrm{Nb} / \mathrm{Cu}$ multilayers (33), and in-plane. Maximum strain values were reported as $-2.5 \% ; 0.6 \%$ in-plane and $1.5 \% ; 5.0 \%$ along the growth direction, for $\mathrm{Nb}$ and $\mathrm{Cu}$, respectively.

The strains in the individual layers of these metallic multilayer systems can be viewed as transforming the crystal structure, as was proposed for the Au/Ni system where a cubic to tetragonal change accompanies an applied biaxial stress.(34) Simulations of atomic structure using Lennard-Jones potentials for two fcc solids with differing lattice constants and binding energies reveal an fcc to hcp transformation for the case of large atomic misfit.(35) A phase transformation within a layer has been observed for $\mathrm{Fe} / \mathrm{Cu}$ multilayers.(36) A $\alpha$ - $\mathrm{Fe}$ to $\gamma$ - $\mathrm{Fe}$ phase transition accompanies the decrease in the layer pair spacing.

\section{MECHANICAL PROPERTIES}

A variety of experimental techniques have been adapted to assess the physical behavior of metallic multilayers. As anticipated from basic concepts of micromechanics, interesting behaviors departing from the bulk are observed for structures with nanometric to subnanometric layer dimensions. Methods to measure physical behavior can be divided into two general categories - non-destructive and deformation testing (which will include the sought after application of enhanced hardness). Techniques and results in each category will be reviewed with respect to common themes, the potential influence of synthesis/processing route on behavior and the general interest in determining fundamental elastic moduli.

\section{Non-Destructive Testing}

Brillouin spectra of metallic superlattices are excited by laser light. The backscattered light is passed through a Fabry-Perot interferometer to analize various wave modes. The phase velocities can then be fitted to an acoustical model to produce the various elastic constants 

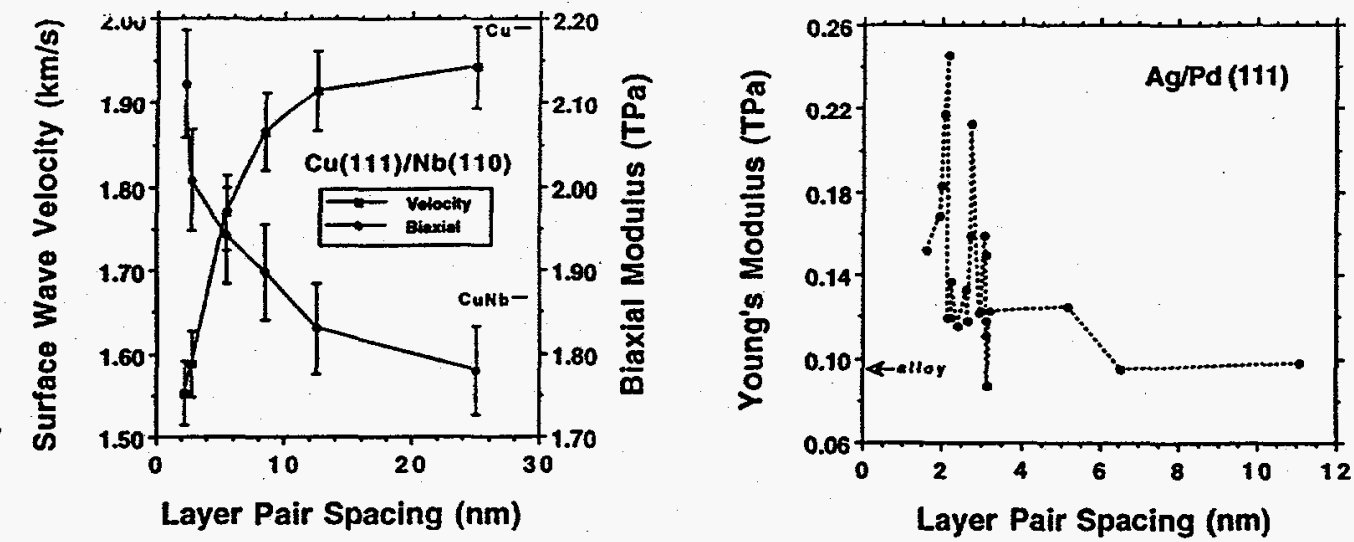

Fig. 5 - The surface wave velocity and biaxial Fig.6 - The Young's modulus of Ag/Pd films as biaixal modulus variations of $\mathrm{Cu} / \mathrm{Nb}$ films.(38) measured using a vibrating reed technique.(46)

$\mathrm{C}_{\mathrm{ij}}$. (37) Preceeding the XRD characterization of the previously described $\mathrm{Nb} / \mathrm{Cu}$ multilayers (32), the surface wave velocities (proportional to $\mathrm{C}_{44}$ ) were found to continuously decrease by $20 \%$ (Fig. 5) as the modulation wavelength decreased to $2 \mathrm{~nm}$.(38) Below this layer pair spacing, no results are reported. This behavior pattern of velocity vs. modulation wavelength occured whether the foils were supported by the substrate or free-standing. Similar findings were reported earlier for $\mathrm{Nb} / \mathrm{Cu}$ deposits on sapphire. $(39,40)$ For these earlier studies, the surface wave velocity was reported to increase below a $2 \mathrm{~nm}$ modulation to the long $(>20 \mathrm{~nm})$ modulation value. Scattering from longitudinal guided modes are determined primarily by $\mathrm{C}_{11}$.(41) The first report of an increase in $\mathrm{C}_{11}$ (and $\mathrm{C}_{55}$ as well) with decreasing modulation wavelength was reported for the $\mathrm{Ag} / \mathrm{Pd}$ system. Samples of composition $\mathrm{Ag} .51 \mathrm{Pd} .49$ were deposited using magnetically enhanced dc triode sputtering. $C_{11}$ increased $20 \%$ above the long $(>10 \mathrm{~nm})$ modulation wavelength value, and $8 \%$ above the rule-of-mixtures value, as the modulation decreased to $1 \mathrm{~nm}$. Apparently contrasting results have been obtained for the $\mathrm{Cu} / \mathrm{Ni}$ system. Brillouin scattering results of ion-beam sputtered samples were characterized with a (111) growth texture and in-plane polycrystallinity.(42) No modulation wavelength dependence was summized. A $0.36 \%$ expansion of the average lattice parameter along the growth direction was reported for modulations $<4.8 \mathrm{~nm}$. This interplanar spacing trend is similar for magnetron sputter deposition of $\mathrm{Cu} / \mathrm{Ni}$ films(43), however the absolute spacings are larger for the ion-beam samples (42), closer to values reported for evaporated $\mathrm{Cu} / \mathrm{NiFe}$ films(34). In another study, single crystal deposits (as verified with RHEED analysis) of $\mathrm{Cu} / \mathrm{Ni}(111)$ were grown on yttriastabilized zirconia (111) substrates by molecular beam epitaxy (MBE).(44) The surface velocity along [110] was measured and interpreted as exhibiting a $60 \%$ enhancement (in $\mathrm{C}_{11}-\mathrm{C}_{12}$ ) as the modulation decreased from $8 \mathrm{~nm}$ to $1 \mathrm{~nm}$. Clearly, there is an effect on lattice structure which differentiates the behavior (and synthesis) of the ion beam deposit from the MBE deposit.

Vibrating film or resonanting beam techniques have been developed to determine several in-plane moduli - biaxial, torsional, Young's and/or flexural. Young's modulus has been determined from the flexural resonant frequency of $\mathrm{Cu} / \mathrm{Ni}$ multilayer films clamped at one end using electrostatic drive and detection near the lateral midpoint of the sample.(45) These $\mathrm{Cu} .5 \mathrm{Ni} .5$ multilayer samples produced by thermal evaporation, on mica substrates heated to $300^{\circ} \mathrm{C}$, exhibited a $100 \%$ increase in the Young's modulus from $0.15-0.20 \mathrm{TPa}$ for a $10 \mathrm{~nm}$ modulation to $0.4 \mathrm{TPa}$ for a $1.6 \mathrm{~nm}$ modulation. Young's modulus (E) measurement of $\mathrm{Ag} / \mathrm{Pd}$ 
foils using the vibrating reed technique (Fig. 6) has shown up to $100 \%$ enhancements for modulations between 1.5 and $5 \mathrm{~nm}$.(46) Ag.5Pd .5 multilayer films were rf sputtered onto thin $\mathrm{Si}(100)$ reed substrates $(15 \mathrm{~mm}$ in length $\times 100 \mu \mathrm{m}$ ) whose temperature did not rise above $310 \mathrm{~K}$ during the deposition process. Less than a $10 \%$ variation in $\mathrm{E}$ could be attributed to thickness nonuniformities. The enhancement of $\mathrm{E}$ takes on a bimodal behavior as a function of $\mathrm{Ag} / \mathrm{Pd}$ modulation wavelength with peaks at 2.2 and $2.8 \mathrm{~nm}$. A reduction in enhancement to the ruleof-mixtures value was observed for samples progressively annealed to $90 \%$ homogenization. The biaxial modulus has been reported from measuring the strain displacement of the frequency of normal modes of a vibrating film.(47) For the previously mentioned $\mathrm{Nb} / \mathrm{Cu}$ sample set (32), the biaxial modulus was found to increase $20 \%$ above the rule-of-mixtures value (approaching that of pure $\mathrm{Cu}$ ) as the modulation wavelength decreased to $2 \mathrm{~nm}$ (Fig. 5).(38) Behavior below the $2 \mathrm{~nm}$ modulation was not reported.

The Debye-Waller factor is a negative exponential proportional to the mean-square displacement of an atom. Determination of the Debye-Waller factor can provide a measure of lattice stiffening. From low-energy electron diffraction measurements of 1-to-10 monolayers of $\mathrm{Cu}$ and $\mathrm{Ni}$, each grown on $\mathrm{Ru}(0001)$ by thermal evaporation, a strong interfacial interaction (by a factor of 2) was found for these pseudomorphic overlayers.(48) The interaction became longranged for overlayer film thicknesses $>10$ monolayers, with a return of the vibrational amplitude

to bulk-like values. The ${ }^{197} \mathrm{Au}$ Mossbauer spectra have been measured for Au/Ni multilayers(49) whose synthesis and structure were previously presented $(25,26)$. From 16 to $75 \mathrm{~K}$, the Debye-Waller factor of Au for a 50 layer pair, $2 \mathrm{~nm}$ modulation is found to be 3 times greater than bulk $\mathrm{Au}$. This increase suggests lattice stiffening hence significant modulus enhancement for the multilayer.

\section{Deformation Testing}

The testing of materials through and beyond the elastic regime, in some cases to failure, requires mechanically loading the sample to very high stress levels. Representative techniques in this testing category used to evaluate elastic moduli in metallic multilayer films are the bulge deflection test and the tensile test. Past and recent examples of each will permit evaluation of development in testing methods and place comparisons of experimental findings in perspective.

The bulge deflection test has been used to measure the biaxial elastic modulus of metallic multilayers. Early findings were characterized by the presence of a non-linear elastic response for strains levels approaching material failure and an enhancement of the elastic modulus as the modulation wavelength, i.e. the layer pair spacing, decreased. $(22,50,51)$ Increases up to $200 \%$ beyond the measured rule-of-mixtures values were obtained, typically with peak behavior near a $2 \mathrm{~nm}$ modulation. Multilayers exhibiting the so-called "supermodulus effect"(51) consisted of noble.5-transition .5 metal combinations of binary alloy systems with continuous solubility, such as $\mathrm{Au}-\mathrm{Ni}, \mathrm{Ag}-\mathrm{Pd}$, and $\mathrm{Cu}-\mathrm{Ni}$. The multilayers were prepared by thermal evaporation on mica substrates held at $200-350^{\circ} \mathrm{C}$ following deposition of an initial seed layer to induce a (111) texture. These growth condition favor formation of a dense columnar deposit, ideal for thin film mechanical studies. Upon annealing the samples, a decay of the enhanced modulus to bulk values accompanied progressive homogenization of the multilayer. The foils were bulge tested by applying gas pressure to a circular mounted, free-standing multilayer foil. The height of the bulge was measured as function of the applied gas pressure. To remove slack, i.e. any wrinkles, from the mounted multilayer foils an initial pressure po (always $<0.2 \mathrm{kPa}$ ) was applied. Two bulge test methods were employed. For the measurements of the Au/Ni multilayer foils, a Bourdon gauge was used to measure applied pressure $( \pm 0.04 \mathrm{kPa})$ and a micrometer to measure 
the total bulge deflection $( \pm 2.5 \mu \mathrm{m})$. For the measurement of $\mathrm{Ag} / \mathrm{Pd}$ and $\mathrm{Cu} / \mathrm{Ni}$, an electrical transducer was used to monitor pressure $( \pm 0.04 \mathrm{kPa})$ and a laser interferometer to provide a more precise measure of the bulge deflection $( \pm 0.3 \mu \mathrm{m})$ beyond po. Although less precise, use of the micrometer provided a total measure of deflection, including the initial height $h_{0}$ associated with $p_{0}$. The stress-strain response of the Au/Ni was typified by a linear regime extending beyond $0.2 \%$ strain, followed by yielding, then failure at strains exceeding $0.4 \%$.(22) A linear regression fit to the initial stress-strain slope produced the biaxial modulus. For enhancements of $35-50 \%$, e.g. a $2.1 \mathrm{~nm}$ modulation compared to a $7.1 \mathrm{~nm}$ modulation, linearity was observed in the stress-strain plots to $0.4 \%$ strain. For the shortest modulation spacings, non-linearity in the stress-strain response extended to smaller strain values, e.g. non-linearity appears beyond $0.1 \%$ strain for a $1.8 \mathrm{~nm}$ modulation which exhibits a $60 \%$ enhancement.(22) For the bulge tests of $\mathrm{Ag} / \mathrm{Pd}$ and $\mathrm{Cu} / \mathrm{Ni}$, the non-linear response extends to strains as small as $0.05 \%$ for all samples. The extent of non-linearity generally increases as the modulation spacing decreases, e.g. a fully homogenized $2.24 \mathrm{~nm}$ Ag/Pd sample (with a zero composition amplitude) exhibits a rule-of-mixtures modulus and is linear to $0.2 \%$ whereas the same sample (with a 0.27 composition amplitude) that shows a $100 \%$ biaxial modulus enhancement is nonlinear above $0.05 \%$ strain.(51) A comparison of typical stress-strain curves cycled between loading and unloading diverge beyond $0.2 \%$ strain, e.g. as for a $2.58 \mathrm{~nm} \mathrm{Ag} / \mathrm{Pd}$ multilayer foil which shows an as-deposited enhancement of 90\%.(51) This indicates that elastic behavior does not extend fully to failure but does so only below $0.2 \%$ strain.

A recent analysis of the bulge test has been proposed to account for the non-linear stressstrain behavior within the elastic regime.(52) A finite element method was developed to model the deformation behavior of a thin film in a bulge test. The finite element model reproduced the deformation behavior as described in an earlier work(53) wherein a closed form solution was determined for the boundary value problem of an initially flat, unstressed, linear elastic membrane clamped over a circular hole. The extent of apparent modulus enhancement was found through the finite element model solution to linearly depend on the initial height $h_{0}$ of the foil which corresponds to the initial pressure $p_{0}$ applied to remove the foil slack (52) The nonlinear elastic behavior beyond small strains becomes linear with $h_{0}$ incorporated into the total foil deflection $h$. As an example, an apparent biaxial enhancement of $25 \%$ could be solely attributed to an $h_{0}=40 \mu \mathrm{m}$. The notion of incorporating an $h_{0}$ correction into the stress-strain results for the $\mathrm{Cu} / \mathrm{Ni}$ and $\mathrm{Ag} / \mathrm{Pd}$ tests $(50,51)$ is plausible since the bulge deflection of these samples was measured with an interferometer. (This scheme of an $h_{0}$ adjustment does not apply to the $75-100 \%$ enhancements measured for the Au/Ni foils since the total bulge height $h$ which incorporates $h_{0}$ was always measured with the micrometer.) The plotting scheme of $(p / h) v s h^{2}$, as adopted to re-evaluate the stress-strain curves(52), was employed to measure the effect of the $h \rightarrow\left(h+h_{0}\right)$ adjustment on the biaxial modulus. Values of $h_{0}$ were interatively fit to produce a linear stress-strain response for the $2.24 \mathrm{~nm} \mathrm{Ag/Pd} \mathrm{multilayer} \mathrm{with} \mathrm{a} 0.27$ composition amplitude(51). To produce a linear stress-strain behavior to strain values of $0.05 \% \rightarrow 0.10 \% \rightarrow$ $0.20 \% \rightarrow 0.40 \%$ requires the following $h_{0}$ values of $0 \mu \mathrm{m} \rightarrow 10 \mu \mathrm{m} \rightarrow 26 \mu \mathrm{m} \rightarrow 42 \mu \mathrm{m}$ creating a corresponding change in the biaxial modulus from $459 \mathrm{GPa} \rightarrow 366 \mathrm{GPa} \rightarrow 268 \mathrm{GPa} \rightarrow 220$ GPa (Fig. 7). The full extent of modulus enhancement can be accounted for if a completely linear stress-strain behavior is generated through the $h_{0}$ correction. This is not reasonable, however, as the onset of non-elastic behavior is found beyond $0.2 \%$ strain. From this guideline, the apparent $100 \%$ enhancement for this $2.24 \mathrm{~nm} \mathrm{Ag/Pd} \mathrm{sample} \mathrm{(51)} \mathrm{would} \mathrm{be} \mathrm{reduced} \mathrm{to} \mathrm{a} 20$ $25 \%$ enhancement. Extending this line of reasoning to the supermodulus findings for the Ag/Pd and $\mathrm{Cu} / \mathrm{Ni}$ foils, initial results for modeling the $h_{0}$ effect reduce the maximum as-deposited 


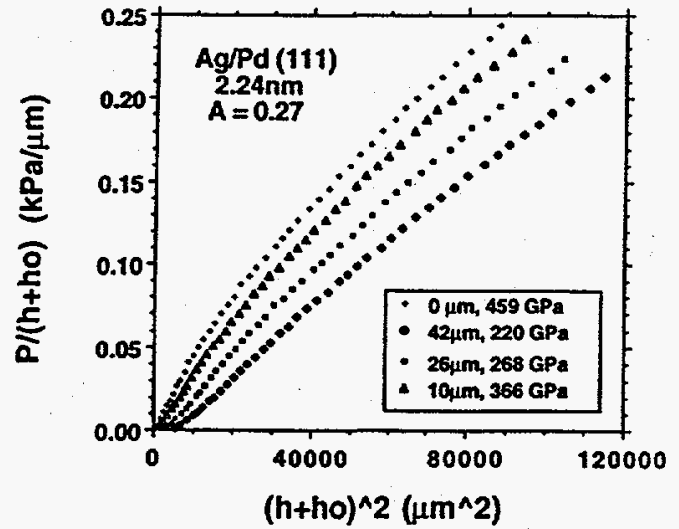

Fig. 7 - Bulge deflection data (51) are adjusted to account for the initial film deflection $h_{0}$.

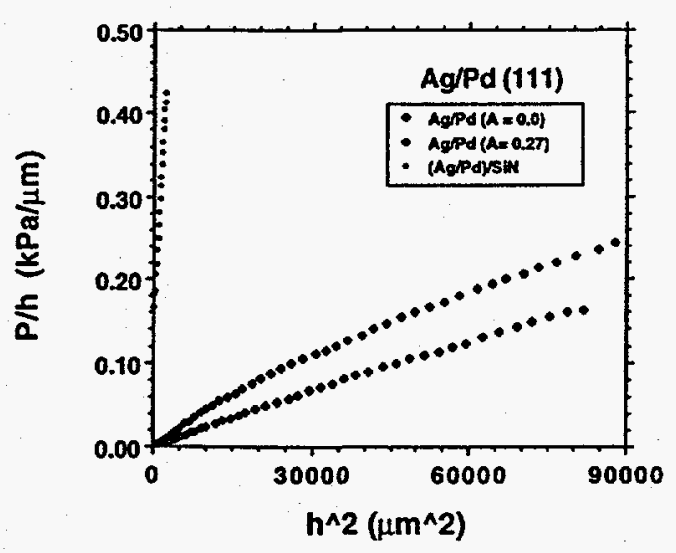

Fig. 8 - Comparison between bulge tests of freestanding(51) vs supported(54) Ag/Pd films.

enhancements from $200-250 \%$ to $75 \%$ or less. Establishing a criteria analysis of normalizing the early bulge test results to a $0.2 \%$ strain follows a traditional treatment of assigning a yield point to apparently non-linear stress-strain results.

A different geometry to measure the deflection of the multilayer film in a bulge test has been developed based on a composite structure.(54) Ag/Pd multilayers are dc magnetron sputter deposited on a low pressure, chemical vapor deposit of $\mathrm{SiN}$ on $\mathrm{Si}(100)$. A Pd epilayer was deposited prior to the multilayer to initiate the (111) texture. By etching through the $\mathrm{Si}$, a thin square, multilayer-support membrane geometry is created. The multilayer and nitride membrane thicknesses are each equal at $0.43 \mu \mathrm{m}$. The use of the support membrane is intended to eliminate the $h_{0}$ problem associated with removing the initial non-planar geometry as encountered for the free-standing multilayer foils at $p_{0}$. A plot of the $(\mathrm{p} / \mathrm{h})$ vs $\mathrm{h}^{2}$ response is linear for the multilayer-membrane composite, as for a $2.5 \mathrm{~nm}$ modulation tested to 2500 $\mu \mathrm{m}^{2}$.(54) The modulus is proportional to slope of the (p/h) vs $\mathrm{h}^{2}$ curves with consideration to the hole size and composite thickness. The multilayer modulus is determined from a composite modulus calculation. No enhancement is reported for the Ag/Pd multilayers as function of modulation wavelength.(54) For comparison to the results for the free-standing foils using a circular geometry requires simultaneous data plotting of bulge test results (Fig. 8). It becomes readily apparent that the small opening for the square geometry and brittle behavior of the SiN membrane place limitations on the value of these test results in any meaningful data comparison. Specifically, nonlinear behavior is reported for the $\mathrm{Ag} / \mathrm{Pd}-\mathrm{SiN}$ films for $\mathrm{h}^{2}$ values within the linear elastic regime of $h_{0}$-uncorrected, free-standing $\mathrm{Ag} / \mathrm{Pd}$ foils. For the free-standing foils (51), the uncorrected $(\mathrm{p} / \mathrm{h}) \mathrm{vs} \mathrm{h}^{2}$ curves are linear to $\mathrm{h}^{2}>10,000 \mu \mathrm{m}^{2}$ whereas the composite $\mathrm{Ag} / \mathrm{Pd}$-SiN moduli(54) are determined for $\mathrm{h}^{2}<2,500 \mu \mathrm{m}^{2}$. Just as important as comparing bulge test results on equal terms is the comparison of microstructure. For the $\mathrm{Ag} / \mathrm{Pd}-\mathrm{SiN}$ samples (54), no superlattice peaks were reported in the XRD spectra for samples with <3nm modulation - which raises concern as to the formation of layering for $<3 \mathrm{~nm}$ modulation samples.

A bimodal enhancement of Young's modulus as a function of modulation wavelength was initially observed for the $\mathrm{Cu} / \mathrm{Ni}$ multilayer system.(55) The multilayers were deposited by evaporation onto mica substrates heated to $\angle 250^{\circ} \mathrm{C}$, following an initial $\mathrm{Cu}$ deposit at $375^{\circ} \mathrm{C}$ which induced the (111) growth texture. Foils were removed from the substrates and tested as rectangular-shaped tensile specimens. Linearity was observed in the stress-strain curves through 
the elastic regime $(>0.4 \%$ strain). Failure of the multilayer tensile specimens typically occured for strains $>0.5 \%$. Calibrated to pure foils of each element as well as a homogeneous alloy deposit, a $50 \%$ modulus enhancement was observed at both the $1.3 \mathrm{~nm}$ and $3 \mathrm{~nm}$ modulation wavelengths.(55) The stress at breaking was maximum for the modulation wavelength intermediate the modulus maxima positions. Using a similar tensile testing technique, Cu/Pd multilayers were examined(56) to evaluate Young's modulus behavior since this system was previously observed to show a biaxial modulus enhancement(22). The Cu/Pd multilayers for tensile testing were deposited by electron beam evaporation onto mica substrates heated to $<60^{\circ} \mathrm{C}$, following an initial $\mathrm{Cu}$ deposit at $300^{\circ} \mathrm{C}$ which induced the (111) growth texture. The low temperature for the multilayer deposit was intended to minimize interface diffusion. Crosssection TEM images revealed highly defected columnar boundaries consistent with the low temperature deposit. Foils were removed from the substrates and tested as rectangular-shaped tensile specimens. Linearity was observed in the stress-strain curves through the elastic regime ( $>0.4 \%$ strain). However, no dependence of Young's modulus on modulation wavelength was detected. In fact, all of the tensile samples displayed a 10-15\% decrease in magnitude below a rule-of-mixtures modulus average. The lack of any apparent modulus enhancement and below rule-of-mixtures result for the Young's modulus may be traced to the low substrate temperature used during the evaporation process. The feature of highly defected columnar boundaries could easily have been the weak link of the structure under tensile load. These evaporated $\mathrm{Cu} / \mathrm{Pd}$ films have a growth morphology similar to the $\mathrm{Au} / \mathrm{Ni}$ samples $(25,26)$ deposited by evaporation on low temperature substrates and analized in the previous section.

Recent tensile tests on magnetron sputtered $\mathrm{Cu} / \mathrm{Ni}$ multilayer films have confirmed Young's modulus enhancement.(43) Unlike the less reliable, (rectangular) tensile-specimen, shape geometry used in earlier studies(55-58), a traditional "dog-bone" shape was prepared by computer-controlled laser cutting.(43) Therefore, analysis of the stress-strain behavior could be assured for only those samples yielding within a well-defined gauge length. The tensile testing of the sputtered $\mathrm{Cu} / \mathrm{Ni}$ proceeded using a $5 \mathrm{~N}$ load cell, at constant strain rate, instrumented to provide load and cross-head displacement. Calibration of elemental and alloy films reproduced bulk values for the Young's modulus of $\mathrm{Cu}, \mathrm{Ni}$ and $\mathrm{CuNi}$. With this experimental procedure, two orders of magnitude more data was recorded per unit strain than for prior tensile studies (55-58). Continuous stress-strain behavior during uniaxial tensile loading was so sensitive as to reveal yield and ultimate stress behavior.(43) A maximum enhancement of 50\% in the Young's modulus was measured for a sputtered $2 \mathrm{~nm}$ multilayer modulation with a possible second local maxima at a $3.8 \mathrm{~nm}$ modulation (Fig. 9). Successive vacuum anneal treatments of the $2 \mathrm{~nm}$ multilayer evidenced progressive decay in the modulus enhancement to the rule-of-mixtures value. Evidence of an atomic microstructure similar to that of the evaporated $\mathrm{Cu} / \mathrm{Ni}$ multilayers(50), which evidenced the "supermodulus" behavior, was revealed by agreement between the measured diffusivities at $400^{\circ} \mathrm{C}$. Tensile testing has been shown to exhibit linear stress-strain behavior in the elastic regime - confirming the exitence of modulus enhancement.

Compression testing has been recently explored to examine the elastic moduli of multilayers. A diamond anvil cell was used to apply high-hydrostatic pressure to a multilayer film while being simultaneously probed with synchrotron radiation in the $\theta / 2 \theta$ mode.(59) The pressure dependence of lattice constants obtained from the peak position of each Bragg reflection offers a means to investigate the compressibility behavior. Measurements were collected simultaneously, then reproduced, of both the Au buffer layer and the $2 \mathrm{~nm} \mathrm{Au} / \mathrm{Ni}$ multilayer film prepared by the previously described evaporation method(25). The compression of the Au buffer layer with applied pressure followed the behavior of bulk Au. However, the $2 \mathrm{~nm} \mathrm{Au} / \mathrm{Ni}$ superlattice reflection appeared anomalously incompressible, by almost an order of magnitude, as compared to bulk values for either $\mathrm{Au}$ or $\mathrm{Ni}$.(59) 


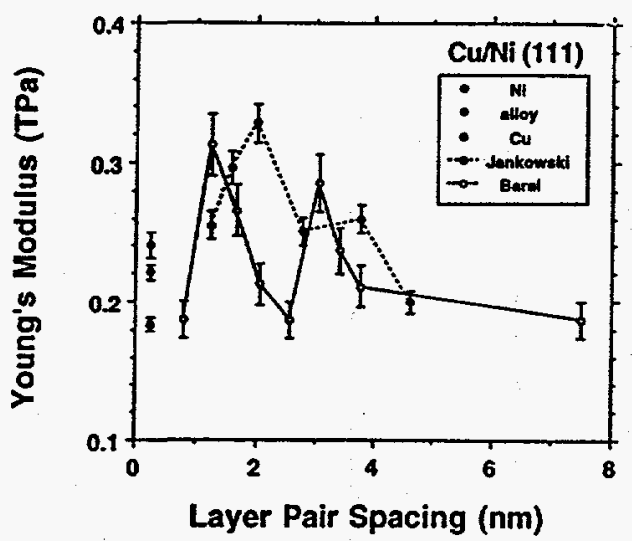

Fig. 9 - Comparison of tensile test results for $\mathrm{Cu} / \mathrm{Ni}$ multilayer films $(43,55)$.

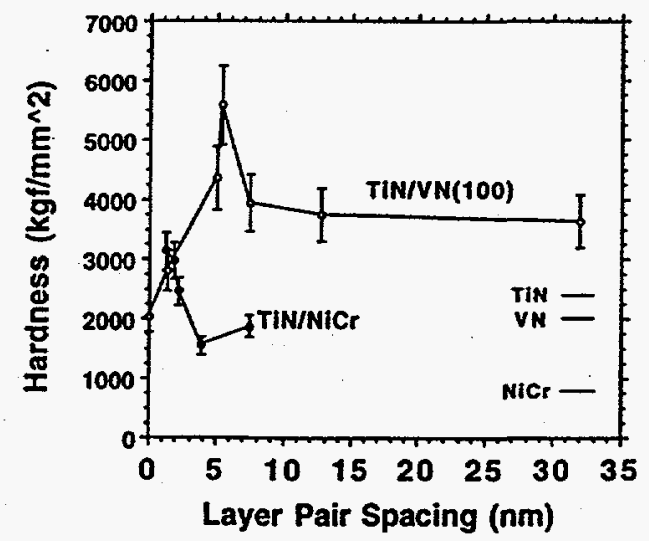

Fig.10- The microhardness of TiN/VN(66) and (TiN). $.7(\mathrm{NiCr}) .3(70)$ multilayer films.

Attempts have been made to measure the elastic moduli of $\mathrm{Au} / \mathrm{Ni}$ multilayer films with a micro-indenter.(60) The Au/Ni multilayers were sputter deposited onto a variety of Si substrate configurations. The indenter moduli of the multilayer films as computed from indentation unloading curves are not directly interpretable with respect to either a shear, biaxial or Young's modulus. The indenter modulus for each multilayer was interpreted as a bulk-like average determined from the contact stiffness of the highly deformed, material region under the indenter. A microbeam deflection study using the microindenter to load and measure the displacement of a composite $\mathrm{Au} / \mathrm{Ni}$ multilayer-Si beam (60) also proved ambiguous. In preparation of the $\mathrm{Si}$ micro-cantilever beams, signficant undercut at the fixed end of the cantilever created an uncertainty as to the actual beam length. Corrections to fit a series of modulus measurements to adjusted beam lengths resulted in increasing the measured film modulus by more than $100 \%$ which is as large or larger than the magnitude of enhancement found for any recent Young's modulus investigation $(43,46)$. Hardness measurements reported for indentation depths greater than $10 \%$ of the film thickness $(60)$ were not representative of the Au/Ni multilayer as later proven in a Meyer-plot analysis of applied load vs indentation depth(61). In all, the microindentation/deflection studies have potential but proved wanton in this case for evaluating the elastic moduli of anisotropic behavior in Au/Ni metallic multilayers.

A property that is useful to assess the metallic multilayer application as protective coatings is the microhardness. Microindentation is most useful to determine the hardness of submicron thick films or coatings $(62,63)$ as indentation depths are controllable to $<10 \mathrm{~nm}$. The microindenter measurement is analogous to a Vickers hardness measurement. Typically, a pyramidal-shaped tip is used to produce indentations at $<10 \%$ the film thickness in order to avoid contribution from the underlying substrate. An observation(64) that dislocation mobility should be inhibited by the composition modulation when combined with layers small enough to cease dislocation generation mechanisms within a given layer implies that the multilayer strength and hardness should increase for decreasing modulation wavelengths(65). Multilayer coatings of $\mathrm{TiC} / \mathrm{TiB}_{2}$ were shown to be more wear protective in cutting steel than either layer compound material.(7) An enhancement of the Vickers microhardness was measured for single crystal TiN/VN(100) superlattices prepared by reactive magnetron sputtering on $\mathrm{MgO}(100)$ substrates heated to $700^{\circ} \mathrm{C}$.(66) A $75 \%$ increase was observed for wavelengths decreasing from $32 \mathrm{~nm}$ to $7.5 \mathrm{~nm}$, at which point the hardness increased an additional $75 \%$ as the wavelength decreased to $5 \mathrm{~nm}$ (Fig. 10). Below $5 \mathrm{~nm}$, the hardnes returned to rule-of-mixtures values. A 
similar behavior for hardness as a function of modulation wavelength was observed for TiN/(V. $\left.{ }_{6} \mathrm{Nb} .4\right) \mathrm{N}$ multilayers(67), TiN/NbN(68) and for TiN/Ti multilayers(69). The hardness of (TiN). $7 /(\mathrm{NiCr}) .3$ multilayers deposited onto tool steels were assessed using a ultramicroindentation system.(70) The 2-3 $\mu \mathrm{m}$ thick coatings were deposited using opposedcathode, reactive unbalanced magnetron sputtering. An Ar- $\mathrm{N}_{2}$ gas mixture was used to initially sputter etch the substrate at high pressure then as a reactive working gas at lower pressure. The sputter deposition process combined with an applied substrate bias led to gradual heating of the substrate to $400^{\circ} \mathrm{C}$. The penetration depth of the indenter was kept below $10 \%$ of the film thickness. The hardness increased to a maximum $60 \%$ above the rule-of-mixtures value as the modulation wavelength decreased to $1.2 \mathrm{~nm}$. Enhanced microhardness has also been measured for the metallic multilayer systems which exhibit modulus enhancement. A progressive increase in hardness to a value $60 \%$ greater than a rule-of-mixtures value was measured for $\mathrm{Cu} / \mathrm{Ni}$ multilayers as the modulation wavelength decreased to $<2 \mathrm{~nm}$.(71) For sputter deposited Au/Ni multilayers, a baseline increase of $50 \%$ above the rule-of-mixtures value was reported with a maximum enhancement of $100 \%$ at a $2 \mathrm{~nm}$ wavelength.(61) The Si substrate was shown to effect the measured film hardness for indentation depths greatcr than $6-8 \%$ of the film thickness.

\section{DISCUSSION}

It is clear, that the deposition conditions greatly impact the structure and properties of multilayer films. Not just between different vapor deposition processes but even as a consequence of the subtle changes within a given method. Comparisons of lattice distortions and physical properties must keep this fundamental issue in mind. For example, the Knoop hardness of TiN coatings varies from $5 \mathrm{GPa}$ to $25 \mathrm{GPa}$ as the composition of the working gas is varied from zero to 50 at.\% $\mathrm{N}_{2}$ during the reactive sputter deposition of $\mathrm{Ti}$ (72).

Differences in physical behavior also arise between metallic multilayers with dissimilar crystal structure and solubility. Examples have been given for $\mathrm{fcc} / \mathrm{fcc}$ systems with continuous solubility (as $\mathrm{Au} / \mathrm{Ni}, \mathrm{Ag} / \mathrm{Pd}$ and $\mathrm{Cu} / \mathrm{Ni}$ which tend to show in-plane stiffening) and for bcc/fcc systems with virtually no solubility (as $\mathrm{Nb} / \mathrm{Cu}$ and $\mathrm{Mo} / \mathrm{Ni}$ which tend to show softening along the growth direction). A general observation has been made that lattice expansion perpendicular to the film plane is accompanied by shear wave softening as the modulation wavelength decreases for bcc/fcc multilayers. (73) The coexistence of in-plane contraction accompanying the expansion along the growth direction has been noted in describing the general physical features that need to be accounted for in modeling the physical behavior of metallic multilayers.(74) Modeling physical behavior incorporating these physical features is clearly desireable as is done in a pseudopotential approach to explain multilayer modulus anomalies based on in-plane lattice contraction. $(28,34,75,76)$ The effect of phase transformations within a multilayer also impact the physical properties. A $\alpha$-Fe to $\gamma$-Fe phase transition accompanies a decrease in the layer pair spacing for $\mathrm{Fe} / \mathrm{Cu}$ multilayers.(36) Whereas the $\mathrm{Cu}$ lattice expands along the growth direction, similar to the $\mathrm{Nb} / \mathrm{Cu}$ case (32), surface phonon velocities measured by Brillouin scattering, indicate the $\mathrm{Fe} / \mathrm{Cu}$ multilayers stiffen as the layer pair spacing decreases.

Surface characterization techniques, as scanning tunneling microscopy, can provide qualitative structural features for topology and reconstruction configurations. However, quantitative use for absolute measures, as for surface roughness, can be misleading.(77) Recent use of the atomic force microscopy for nanoindentation has shown the sensitivity to differentiate between the elastic moduli of native oxide layers and the underlying bulk for refractory metal, single crystals.(78) The use of these microscopy techniques should continue to prove useful as the dimension of the probe is on the same scale as the controlling layer dimensions. 
The emphasis of this review has been on the experimental results for the synthesis, structure and mechanical properties of metallic multilayers. Predictive modeling of elastic moduli based on selected observations, as incoherent lattice expansion (79-83), do not prove useful in explaining the nature of the broad range of real physical behavior. Synthesis experiments and theoretical explanations can be complimented for examining the observed physical behaviors. For example, shear wave softening of $\mathrm{Mo} / \mathrm{Ni}$ multilayers designed with non-periodic layer thicknesses at small modulation wavelengths leads to the conclusion that an electron mechanism based on mini-zone boundaries is not responsible for the observed behavior.(84) This physical behavior is suggested as electronic effects which lead to singularities in the variation of the elastic constants with modulation wavelength are predicted not to make contribution to the elastic properties in real systems.(85) The dynamical effects of biaxial strain in short period $\mathrm{Cu} / \mathrm{Ni}(111)$ superlattices were calculated within the framework of a central force-constant model and a surface Green function matching technique.(86) Spectral features of the metallic superlattice, i.e. dispersion relations involving extended modes, indicate a general stiffening with strain. Approaches as these should be useful in further assessing the mechanisms of the anomalous properties of metallic multilayers.

\section{SUMMARY}

Deposition growth conditions are shown to effect the structure and properties of metallic multilayers at the nanoscale. On average, lattice expansion is measured normal to the film plane and lattice contraction within the film plane. In some instances, individual layers exhibit a nonPoisson effect, e.g. lattice contraction in the film plane and along the growth direction. Lattice stiffening from interfacial strains typically yields shear softening while enhancing moduli inplane. The inhibition of dislocation mobility by composition modulation enhances strength and hardness. Limitations and advantages exist and must be accepted for each method of synthesis and characteriztion of both structure and properties.

\section{ACKNOWLEDGMENTS}

This work was performed under the auspices of the United States Department of Energy by Lawrence Livermore National Laboratory under contract \#W-7405-Eng-48.

\section{REFERENCES}

1. S. Hashimoto, Y. Ochiai and K. Aso, J. Appl. Phys. 66, 4909 (1989).

2. S. Hashimoto, Y. Ochiai and K. Aso, J. Appl. Phys. 67, 2136 (1990).

3. E. Devlin, V. Psycharis, A. Kostikas, A. Simopoulos, D. Niarchos, A. Jankowski, T. Tsakalakos, H. Wan and G. Hadjipanayis, J. Magn. Magn. Mater. 120, 236 (1993).

4. H.He, C.Lee, F.Lamelas, W.Vavra, D.Barlett and R.Clarke, J. Appl. Phys. 67, 5412 (1990).

5. L. Wu, N. Nakayama, B. Engel, T. Shinjo and C. Falco, Jpn. J. Appl. Phys. 32, 4726 (1993).

6. A. Jankowski, D. Makowiecki, M. Wall and M. McKernan, J. Appl. Phys. 65, 4450 (1989).

7. H. Holleck, C. Kuhl and H. Schulz, J. Vac. Sci. Technol. A 3, 2345 (1985).

8. M. Niibe, M. Hayashida, T. Iizuka, A. Miyaku, Y. Watanabe, R. Takahashi and Y. Fukdo, SPIE Proc. 1343, 2 (1990).

9. M. Stearns, C. Chang and D. Stearns, J. Appl. Phys. 71,187 (1992).

10 D. Stearns, R. Rosen and S. Vernon, J. Vac. Sci. Technol, A 9, 2662 (1991). 
11. A. Jankowski, Mater. Sci. Eng'g. B 6, 191 (1990).

12. H. Winters, H. Coofal and W. Eckstein, J. Vac. Sci. Technol. A 11, 657 (1993).

13. R.E. Somekh, J. Vac. Sci. Technol. A 2, 1285 (1984).

14. W.D. Westwood, Mater. Res. Soc. Bull. 13 (12), 46 (1988).

15. W.D. Westwood, J. Vac. Sci. Technol. 15, 1 (1978).

16. A. Jankowski, Thin Solid Films 220, 166 (1992).

17. I. Petrov, I. Ivanov, V. Orlinov and J. Sundgren, J. Vac. Sci. Technol. A 11, 2733 (1993).

18. W. Bennett, J. Leavitt and C. Falso, Phys. Rev B $\underline{35}, 4199$ (1987).

19. A. Segmuller, I. Noyan and V. Speriosu, Prog. Cryst. Growth Charact. 18, 21 (1989).

20. J. Sundgren, J. Birch, G. Hakansson, L. Hultman and U. Helmersson, Thin Solid Films 193/194, 818 (1990).

21. Z. Xu, Z. Tang, S. Kevan, T. Novet and D. Johnson, J. Appl. Phys. 74, 905 (1993).

22. W. Yang, T. Tsakalakos and J. Hilliard, J. Appl. Phys. 48 , 876 (1977).

23. D. deFontaine, private communication to J. Hilliard (1980).

24. I. Schuller and A. Rahman, Phys. Rev. Lett. 50, 1377 (1983).

25. H. Dohnomae, N. Nakayama and T. Shinjo, Mat. Trans. Jpn. Instit. Metals 31, 615 (1990).

26. N. Nakayama, L. Wu, H. Dohnomae, T. Shinjo, J. Kim and C. Falco, J. Magn. Magn. Mater. 126, 71 (1993).

27. A. Jankowski, J. Appl. Phys. 71, 1782 (1992).

28. A. Jankowski, J. Phys. Chem Sol. 50, 641 (1989).

29. A. Jankowski, Superlatt. Microstruc. 6 , 427 (1989).

30. J. Chaudhuri, V. Gondhlekar and A. Jankowski, J. Appl. Phys. 71, 3816 (1992).

31. J. Chaudhuri, S.M. Alyan and A.F. Jankowski, Thin Solid Films 219, 63 (1992).

32. A. Fartash, M. Grimsditch, E. Fullerton and I. Schuller, Phys. Rev. B 47, 12813 (1993).

33. I. Schuller and M. Grimsditch, J. Vac. Sci. Technol. B 4, 1444 (1986).

34. A. Jankowski and T. Tsakalakos, J. Phys. F: Met. Phys. 15, 1279 (1985).

35. J. Hoekstra, H. Yan, G. Kalonji and H. Jonsson, J. Mater. Res. 2, 2190 (1994).

36. E. Fullerton, I. Schuller, F. Parker, K. Svinarich, G. Eesley, R. Bhadra and M. Grimsditch, J. Appl. Phys. 73, 7370 (1993).

37. M. Grimsditch, Phys. Rev. B 31, 6818 (1985).

38. A. Fartash, E. Fullerton, I. Schuller, S. Bobbin, J. Wagner, R. Cammarata, S. Kumar and M. Grimsditch, Phys. Rev. B 44, 13760 (1991).

39. A. Kueny, M. Grimsditch, K. Miyano, I. Banerjee, C. Falco and I. Schuller, Phys. Rev. Lett. $\underline{48}, 166$ (1982).

40. R. Zanoni, J. Bell, G. Stegeman and C. Seaton, Thin Solid Films 154, 225 (1987).

41. J. Dutcher, S. Lee, J. Kim, G. Stegeman and C. Falco, Phys. Rev. Lett. 65, 1231 (1990).

42. S. Kumar, R. Bhadra, A. Fartash, M. Grimsditch, C. Kim, S. Qadri and A. Edelstein, Phys. Rev. B 44, 5905 (1991).

43. A. Jankowski, E. Sedillo and J. Hayes, Jpn. J. Appl. Phys. 33, 4511 (1994).

44. K. Sakaue, K. Kamigaki, M. Niboshi, T. Nagahara, N. Sano, H. Terauchi and A. Yoshihara, J. Magn. Magn. Mater. 126, 207 (1993).

45. L. Testardi, R. Willens, J. Krause, D. McWhan and S. Nakahara, J. Appl. Phys. 52, 510 (1981).

46. H. Mizubayashi, T. Yamaguchi, W. Song, A. Yamaguchi and R. Yamamoto, J. Magn. Magn. Mater. 126, 127 (1993).

47. A. Fartash, I. Schuller and M. Grimsditch, Rev. Sci. Instrum. 62, 494 (1991). 
48. D. O'Neill and J. Houston, Phys. Rev. B 42, 2792 (1990).

49. S. Nasu, Y. Kobayashi, T. Shibatani, H. Dohnomae N. Mima and T. Shinjo, J. Magn. Magn. Mater. 126, 231 (1993).

50. T. Tsakalakos and J. Hilliard, J. Appl. Phys. 54, 734 (1983).

51. G. Henein and J. Hilliard, J. Appl. Phys. 54, 728 (1983).

52. M. Small and W. Nix, J. Mater. Res. Z, 1553 (1992).

53. T. Tsakalakos, Thin Solid Films 75, 293 (1981).

54. M. Small, B. Daniels, B. Clemens and W. Nix, J. Mater. Res. 2, 25 (1994).

55. D. Baral, J. Ketterson and J. Hilliard, J. Appl. Phys. 57, 1076 (1985).

56. B. Davis, D. Li, D. Seidman, J. Ketterson, R. Bhadra and M. Grimsditch, J. Mater. Res. 7, 1356 (1992).

57. A. Jankowski and T. Tsakalakos, Scripta Metal. 19, 625 (1985).

58. A. Jankowski and T.Tsakalakos, J. Appl. Phys. 57, 1835 (1985).

59. Y.Fujii, Y.Ohishi, H.Konishi, N.Nakayama, T.Shinjo, J.Magn.Magn.Mater. 126,192(1993).

60. S. Baker, A. Jankowski, S. Hong and W. Nix, Mat. Res. Soc. Symp. Proc. 188, 289 (1990).

61. A. Jankowski, J. Magn. Magn Mater. 126, 185 (1994).

62. M. Doerner and W. Nix, J. Mater. Res. 1, 601 (1986).

63. W. Oliver and G. Pharr, J. Mater. Res. I, 1564 (1992).

64. J. Koehler, Phys. Rev. B 2, 547 (1970).

65. L. Palatnik, A. Il'inskii and N. Sapelkin, Sov. Phys. Sol. Stat. $\underline{8}, 2016$ (1967).

66. U. Helmersson, S. Todorova, L. Market, S. Barnett, J. Sundgren and J. Greene, J. Appl. Phys. 62, 481 (1987).

67. K. Hubbard, T. Jervis, P. Mirkarimi and S. Barnett, J. Appl. Phys. 72, 4466 (1992).

68. X.Chu, M.Wong, W.Sproul, S.Rohde and S.Barnett, J.Vac.Sci.Technol.A 10, 1604 (1992).

69. K. Shih and D. Dove, Appl. Phys. Lett. 61, 654 (1992).

70. X. Chu, M. Wong, W. Sproul and S. Barnett, Surf. Coatings Technol. 61, 251 (1993).

71. R.Cammarata, T.Schlesinger, C.Kim, S.Qadri, A.Edelstein, Appl.Phys.Lett. 56,1862(1990).

72. F. Elstner, A. Ehrlich, H. Giegengack, H. Kopfer, F. Richter, J. Vac. Sci. Technol. A 12, 476 (1994).

73. B. Clemens and G. Eesley, Phys. Rev. Lett. 61, 2356 (1988).

74. A. Jankowski, Mater. Sci. Eng'g. A 114, L17 (1989).

75. A. Jankowski, J. Phys. F: Met. Phys. 18,413 (1988).

76. A. Jankowski and T. Tsakalakos, Mater. Sci. Eng'g. B 6, 87 (1990).

77. E. Tekman and S. Ciraci, Phys. Rev. B 40, (1989).

78. S. Hues, C. Draper and R. Colton, J. Vac. Sci. Technol. B 12, 2211 (1994).

79. D. Wolf and J. Lutsko, J. Mater. Res. 4, 1427 (1989).

80. J. Jaszczak, S. Phillpot and D. Wolf, J. Appl. Phys. 68, 4573 (1990).

81. R. Jones, J. Slotwinski and J. Mintmire, Phys. Rev. B 45, 13624 (1992).

82. C. Gilmore and V. Provenzano, Phys. Rev. B 42, 6899 (1990).

83. R. Cammarata and K. Sieradzki, Phys. Rev. Lett. 62, 2005 (1989).

84. G. Richardson, J. Makous, H. Yu and A. Edelstein, Phys. Rev. B 45, 12114 (1992).

85. J. Chang and M. Stott, Phys. Rev. B 46, 10423 (1992).

86. L. Colombo, L. Miglio, R. Brito-Orta, V. Velasco and F. Garcia-Moliner, J. Appl. Phys. 70, 2079 (1991). 